\title{
Security-Constrained Optimal Protection Coordination for Dual-Setting Digital Directional Overcurrent Relays in the Distribution Network Including Non-Renewable/Renewable Synchronous Distributed Generation
}

\author{
Sasan Pirouzi \\ Semirom Branch, Islamic Azad University \\ Hosein Hasan Shahi \\ Marvdasht Branch, Islamic Azad University \\ Mohammad Reza Akbai Zadeh \\ Technical and Vocational University \\ Amirreza Naderipour ( $\square$ namirreza@utm.my ) \\ University of Technology Malaysia \\ Zulkurnain Abdul-Malek \\ University of Technology Malaysia
}

\section{Research Article}

Keywords: Distribution network, Dual setting digital directional overcurrent relay, Relay unavailability, Security-constrained optimal protection coordination, Synchronous distributed generation

Posted Date: February 26th, 2021

DOI: https://doi.org/10.21203/rs.3.rs-238081/v1

License: (c) (i) This work is licensed under a Creative Commons Attribution 4.0 International License.

Read Full License 
1 Security-Constrained Optimal Protection Coordination for Dual-Setting Digital

\section{Directional Overcurrent Relays in the Distribution Network Including Non-} Renewable/Renewable Synchronous Distributed Generation

$6 \quad{ }^{1}$ Power System Group, Semirom Branch, Islamic Azad University, Semirom, Iran

$7 \quad{ }^{2}$ Department of Electrical Engineering, Marvdasht Branch, Islamic Azad University, Marvdasht, Iran

$8{ }^{3}$ Department of Electrical and Computer Engineering, Faculty of Bahonar, Technical and Vocational University (TVU), Shiraz, Iran

$9{ }^{4}$ Institute of High Voltage \& High Current, School of Electrical Engineering, Faculty of Engineering, Universiti Teknologi Malaysia, Johor

10 Bahru, 81310, Malaysia

11 *Corresponding Author: namirreza@utm.my

12

13 Abstract: In this paper, the security-constrained optimal protection coordination (SCOPC) is introduced for dual setting digital

14 directional overcurrent relay (DDOCR) in distribution network, which including renewable and non-renewable synchronous 15 distributed generation (SDG). The SCOPC minimizes the total operation time of DDOCRs in primary and backup protection 16 operating to achieve a fast protection coordination. Also, to improve the flexibility in DDOCRs setting, the allowable limits of A 17 and B coefficients, pickup current (PC) and time dial setting (TDS) in both reverse and forward directions are considered as 18 constraints. Another constraint is the Coordination Time interval (CTI). To consideration of the mentioned scheme security, the 19 SCOPC mechanism considered the unavailability of DDOCRs due to their failure, so the stochastic method is used to modelling 20 of this parameter. To calculate the fault current, network variables are proportional to the daily stochastic operation results of 21 distribution network. Moreover, the proposed problem is implemented on the standard distribution networks, and then the optimal 22 solution is obtained with hybrid algorithm of grey wolf optimization (GWO) and training and learning optimization (TLBO). The 23 numerical results illustrate that the proposed algorithm is able to achieve a reliable and fast protection coordination that has a low 24 standard deviation.

Index Terms: Distribution network, Dual setting digital directional overcurrent relay, Relay unavailability, Security-constrained optimal protection coordination, Synchronous distributed generation. 
$b \cdot \psi_{B} \quad$ Indices and sets of DDOCRs in backup protection mode

$f \cdot \psi_{f} \quad$ Indices and sets of faults

$g \cdot \psi_{D G} \cdot \psi_{R E S} \quad$ Indices and sets of SDGs, sets of renewable SDGs

$h \cdot \psi_{H} \quad$ Indices and sets hours

$k(f) \quad$ Fault location for " $f$ ” faults

$n \cdot \psi_{N} \quad$ Indices and sets of buses

$r \cdot \psi_{R} \quad$ Indices and sets of DDOCRs

Indices and sets of scenario samples due to uncertainty of load, renewable power, energy

$s \cdot \psi_{S C 2}$ price

$w \cdot \psi_{S C l} \quad$ Indices and sets of DDOCR unavailability uncertainty scenarios

36

37 2) Variables
$A^{f w / p}, A^{r v / b}$
Constant coefficient of DDOCR in forward and reverse discretion
$B^{f w / p}, B^{r v / b}$
Constant coefficient of DDOCR in forward and reverse discretion
$I^{F}$
Fault current flow in distribution line in per unit (p.u.)
$I P^{f w / p}, I P^{r v / b}$
Pickup current of DDOCR in forward and reverse discretion
$I^{S C}$
Fault current flow in DDOCR (p.u.)
$O C$
Expected operation cost $(\$)$
OT
Expected total operation time of all DDOCRs (sec)
$P^{D G}, Q^{D G}$
Active and reactive power of SDG (p.u.)
$P^{L}, Q^{L}$
Active and reactive power of distribution line (p.u.)
$P^{S}, Q^{S}$
Active and reactive power of distribution substation (p.u.)
$T D S^{f w / p}, T D S^{r v / l}$
Time dial setting of DDOCR in forward and reverse discretion (sec)
$t^{f w / p}, t^{r v / b}$
Operation time of DDOCR in forward and reverse discretion (sec)
$V, \delta$
Voltage magnitude (p.u.) and voltage angle (rad)
$V^{F}$
The voltage after fault occurrence (p.u.)
$\mu$
Lagrange multipliers

3) Constants: 
$A^{G}$

$A^{L}$

$A^{\min }, A^{\max }$

$B^{L}, G^{L}$

$B^{\min }, B^{\max }$

CTI

$I P^{\min }, I P^{\max }$

$P^{D}, Q^{D}$

$\bar{P}^{D G}$

$\bar{Q}^{D G}, \underline{Q}^{D G}$

$\bar{S}^{L}$

$\bar{S}^{S}$

$T D S^{\min }, T D S^{\max }$

\section{$u$}

$\bar{V}, \underline{V}$

$\pi$

$\lambda$

$\beta$

Incidence matrix of SDGs and buses

Incidence matrix of buses and distribution lines with consideration of current direct

Minimum and maximum amount of A constant in DDOCR

Susceptance and conductance of distribution line (p.u)

Minimum and maximum amount of B constant in DDOCR

Coordination time interval (sec)

Upper and lower amount of DDOCR pickup current (p.u.)

Active and reactive load (p.u.)

Maximum amount of generation active power of SDG

Minimum and maximum amount of generation reactive power of SDG (p.u.)

Maximum amount of apparent power of distribution line (p.u.)

Maximum amount of apparent power of distribution substation (p.u.)

Minimum and maximum amount of TDS for DDOCR

DDOCR unavailability

Maximum and minimum amount of voltage magnitude (p.u.)

Probability of scenario occurrence

Energy price $(\$ / \mathrm{MWh})$

Fuel price of non-renewable SDG (\$/MWh)

\section{1. Introduction}

42 According to the U.S. Department of Energy, more than 80 precent of power system faults occur in the distribution network

43 1. Therefore, the implementation of protection coordination for overcurrent relays will be able to achieve the high and fast 44 protection in the distribution network in the fault occurrence conditions and short circuit current fault ${ }^{2}$. However, it should be 45 noted that the presence of synchronous distributed generations (SDGs) in the distribution network, along with environmental 46 and distribution system operation benefits, will cause protection issues. So that, the injected short circuit current level of it is 47 high in the fault occurrence conditions, and leads to loss of protection coordination in the network ${ }^{3}$. To overcome of this issue, 48 different solutions have been proposed that one of them is the use of a fault current limiter (FCL) besides the SDG ${ }^{4}$. This method 49 is suitable for the restoration of protection coordination in the radial distribution network, but since the presence of SDG in the 50 network causes a bi-directional power flow, so it is necessary to use of the optimal protection coordination (OPC) scheme ${ }^{5}$ for 51 digital directional overcurrent relays (DDOCRs) ${ }^{6}$. 
So far, many studies have been conducted on the OPC for directional overcurrent relays (DOCRs) in the distribution network

$53{ }^{7}$. In ${ }^{8}$, the OPC scheme is used for DOCRs in the distribution network, which considers the transient stability limitation as a 54 linear constraint. Based on the obtained results in ${ }^{8}$, it can be seen that the OPC scheme is able to determine the scheduling of 55 DOCRs in such a way that the transient stability limitation is satisfied in the distribution network. In ${ }^{9}$, the OPC mechanism is applied for DOCRs in meshed distribution network that its optimal solution is achieved with a modified particle swarm optimization (PSO) algorithm. According to the results of this paper, the presence of distributed generations (DGs) causes the loss of optimal coordination of DOCRs, so $\operatorname{Ref}^{9}$ has used FCL with optimal size and location to improve or restore the protection coordination. Also, the proposed scheme in ${ }^{9}$ is applied for overcurrent relays (OCRs) in a microgrid (MG) based on work of ${ }^{10}$, but in ${ }^{10}$ the directional FCL (DFCL) is used to restore the protection coordination. The effects of changing in network topology 61 and transformer tap on the OPC of DOCRs have also been investigated in ${ }^{11}$. Also, the OPC problem of relays in the distribution network with a high penetration of DGs has been presented in ${ }^{12}$, which the sine cosine algorithm is used to achieve the optimal solution. Furthermore, the proposed OPC scheme in ${ }^{12}$ is used in ${ }^{13}$, which the whale optimization algorithm is applied to achieve 64 the optimal solution. In ${ }^{14}$, DGs transient stability-constrained traditional protection coordination scheme is provided which is not defined as an optimization problem and uses the existing rules on protection coordination, but note that this scheme can be used for small and radial networks. In ${ }^{15}$, the protection coordination problem of MG is introduced with DOCRs in the presence of FCLs, which the combination of cuckoo search algorithm (CSA) and linear programming (LP) is used to solve it. The proposed framework in ${ }^{15}$ is used in ${ }^{16}$ for distribution network concluding superconducting FCLs (SFCLs) and voltage compensators.

69 Finally, Ref ${ }^{17}$ examined the effects of Inverter-based DGs on the protection coordination of DOCRs. Based on the obtained 70 results of it, these types of DGs are able to quickly interrupt their injected fault current into the network, so Inverter-based DGs 71 don't have the same negative effects as SDGs on the protection coordination of distribution network.

72 According to the state of art of this paper, the following research gaps can be extracted:

73 - In most studies, pickup current (PC) and time dial setting (TDS) are generally considered as DOCR adjustment parameters ${ }^{18-20}$. It is estimated that by adjusting the A and B constants in the digital relays beside the PC and TDS regulations, faster protection coordination can be achieved. In this case, the flexibility of relays setting will be desirable, which has been less studied in other researches.

- In order to evaluate the high security of the OPC scheme, it is necessary to consider the unavailability of relays that are caused by their failure. However, in most works, this issue has not been considered ${ }^{18-20}$, and only the backup relays have been used for the primary relay.

- It is worth noting that in most paper, the results of power flow are used to calculate the fault current generally, and the level of generation power of SDGs in these conditions is considered constant. However, it should be said that the level of generation power of renewable SDGs, such as wind turbine varies according to the weather conditions. SDGs also operate 
at optimal operating conditions. Therefore, it is possible that the calculated fault current by the power flow doesn't to obtain a reliable result.

- Most research has used traditional evolutionary algorithms such as genetic algorithm (GA) and PSO algorithm to solve the OPC problem ${ }^{19}$. It is noteworthy that these algorithms are suitable for simple problems. But the OPC problem considering the A, B, PC and TDS for dual setting DDOCRs, will be a complex problem, which is more evident in larger distribution networks. Therefore, it is anticipated that traditional evolutionary algorithms will not be able to provide a reliable optimal solution in this situation.

To overcome the research gaps that mentioned in this paper, the security-constrained optimal protection coordination (SCOPC) scheme for dual setting DDOCRs in the distribution network in the presence of SDGs is expressed. The proposed problem minimizes the total operation time of all DDOCRs in backup and primary protection modes, while it constrained to the allowable limit of relay adjustment parameters and coordination time interval (CTI). In this mechanism, in order to improve the flexibility of relays adjustments and fast protection coordination achievement in case of fault condition, the proposed scheme achieves the optimal value for A, B, PC and TDS parameters in both forward and reverse directions of DDOCRs. In addition, in order to improve and increase the security of the OPC scheme, the unavailability of relays due to their failure is also considered in the proposed scheme. This parameter is behaved as uncertainty, so the stochastic programming is used to model it. In this method, the Monte Carlo simulation (MCS), proportional to the Bernoulli probability distribution function (PDF), obtains the number of scenarios for the unavailability of the relays uncertainty, which is proportional to the forced outage rate (FOR) of the relays. Moreover, the fault current flow of the relays is calculated in commensurate to the optimal daily operation results of the distribution network in the presence of renewable and non-renewable SDGs. This problem minimizes the expected total operation cost of the distribution network and SDGs, which is limited to the AC power flow equations and the SDG constraints. In this problem, the load consumption, energy price and generation power of renewable SDGs are uncertain, and stochastic programming is applied to model these parameters. In this programing, the MCS method first generates a high number of scenarios in proportion to the Normal/Weibull PDF for the consumed load and the energy price / the production power of the renewable SDGs uncertainties. Then, the Kantorovich method is used as a scenario reduction technique to achieve a low number of scenarios with a high probability of occurrence. In the following, the buses voltage and the current flow through the distribution lines are calculated per different fault locations according to the obtained results of the distribution network optimal operation. Finally, to consider the worst case scenario, at all simulation hours and in all scenarios, the fault current flow through the relay is considered to be equal to the maximum value of the fault current passing through the distribution line where the relay is located. It is noteworthy that the two mentioned problems are nonlinear, which in this paper to achieve a optimal solution including low standard deviation in responding, a hybrid optimization algorithm that is combination of the grey wolf optimization (GWO) and teaching-learning-based optimization (TLBO) algorithms is used. Finally, the novelties of proposed mechanism are as follows: 
- Implementation of the SCOPC scheme in the distribution network considering the uncertainty of the unavailability of dual

115 setting DDOCRs

116 - Flexibility improvement of dual setting DDOCRs and fast protection coordination achievement by considering the

117 adjustment of A, B, PC and TDS parameters in forward and reverse directions of DDOCRs.

118 - Calculate the fault current flow through the relays in proportion to the results of the optimal daily operation of the distribution 119 network.

120 - Apply the combined GWO and TLBO algorithm for the proposed problems to obtain an optimal solution containing low $121 \quad$ standard deviation in responding.

122 The rest of the paper is organized as follows: the SCOPC scheme is described in Section 2. Section 3 presents the problem 123 solution process using a combined GWO and TLBO algorithm. Then the numerical results and conclusion are shown in Sections 1244 and 5, respectively.

\section{Proposed protection coordination mechanism}

In this section, the SCOPC for dual setting DDOCRs in distribution network with SDGs including micro turbines, diesel generators and wind turbine units is presented. In this scheme, the optimal value of the DDOCR adjustment parameters, i.e. $A^{f w / p}$, $A^{r v / b}, B^{f w / p}, B^{r v / b}, I P^{f w / p}, I P^{r v / b}, T D S^{f w / p}$ and $T D S^{r v / b}$, are calculated in a way that the total operation time of these relays is in two

130 primary and backup protection modes is minimized, while the constraint of the coordination time interval (CTI) is satisfied. One 131 of the ability of the proposed SCOPC in this paper is the unavailability of one of mentioned relays that due to their failure, which

132 will be an innovation aspect of this paper. Therefore, the proposed scheme will be modeled as follows:

$$
\min \quad O T=\sum_{w \in \Psi_{s G_{1}}} \pi_{w}\left(\sum_{f \in \Psi_{F}}\left(\sum_{r \in \Psi_{R}} t_{r, f, w}^{f w / p}+\sum_{b \in \Psi_{B}} t_{r, f, b, w}^{r v / b}\right)\right)
$$

Subject to:

$$
\begin{aligned}
& t_{r, f, w}^{f w / p}=T D S_{r}^{f w / p}\left(\frac{A_{r}^{f w / p}}{\left(\frac{I_{r, f}^{S C}}{I P_{r}^{f w / p}}\right)^{B_{r}^{f r / p}}-1}\right) u_{r, w} \quad \forall r, f, w \\
& t_{r, f, b, w}^{r v / b}=T D S_{r}^{r v / b}\left(\frac{A_{r}^{r v / b}}{\left(\frac{I_{r, f, b}^{S C}}{I P_{r}^{r v / b}}\right)^{B_{r}^{r / l b}}-1}\right) u_{r, w} \quad \forall r, f, b, w \\
& A^{\min } \leq A_{r}^{f w / p} \leq A^{\max } \quad \forall r
\end{aligned}
$$




$$
\begin{aligned}
& A^{\min } \leq A_{r}^{r v / b} \leq A^{\max } \quad \forall r \\
& B^{\min } \leq B_{r}^{f w / p} \leq B^{\max } \quad \forall r \\
& B^{\min } \leq B_{r}^{r v / b} \leq B^{\max } \quad \forall r \\
& T D S^{\min } \leq T D S_{r}^{f w / p} \leq T D S^{\max } \quad \forall r \\
& T D S^{\min } \leq T D S_{r}^{r v / b} \leq T D S^{\max } \quad \forall r \\
& I P_{r}^{\min } \leq I P_{r}^{f w / p} \leq I P_{r}^{\max } \quad \forall r \\
& I P_{r}^{\min } \leq I P_{r}^{r v / b} \leq I P_{r}^{\max } \quad \forall r \\
& t_{r, f, b, w}^{r v / b}-t_{r, f, w}^{f w / p} \geq C T I \quad \forall r, f, b, w
\end{aligned}
$$

133 The proposed SCOPC objective function is shown in Eq. (1), which formulates the minimization of the total operation time 134 of DDOCRs in both primary and backup protection operating considering a number of fault locations. Also, the operation time 135 of each relay in the two forward and reverse directions, which correspond to the primary and backup protection modes can be 136 calculated from the constraints in Eq. (2) and (3), respectively ${ }^{3,11}$. In constraints (4)-(11), constraints for DDOCRs adjustment 137 variables, i.e. $A^{f w / p}, A^{r v / b}, B^{f w / p}, B^{r v / b}, I P^{f w / p}, I P^{r v / b}, T D S^{f w / p}$ and $T D S^{r v / b}$, are presented, but it should be noted that in most studies, 138 the values of $\mathrm{A}$ and $\mathrm{B}$ parameters are generally constant for dual setting DOCRs ${ }^{17}$. In this paper, in order to flexibility 139 achievement in the settings of the mentioned relays and attain a faster protection coordination, the number of more adjustment 140 parameters are considered for a dual setting DDOCR relay, which is another innovation for this paper. Finally, the constraint of 141 the coordination time interval (CTI) is provided in constraint (12) ${ }^{8}$. According to the constraint in Eq. (12), the minimum 142 operation time of a relay in the backup protection mode than the relay in the primary protection mode should be more than CTI.

143 In the proposed SCOPC scheme, the unavailability parameter of a DDOCR $(u)$ is used to evaluate the reliability of this scheme.

144 The value of this parameter is equal to zero or one, which is the value of zero/one corresponding to no responding/responding of 145 the DDOCR in the distribution network. This parameter is an uncertainty that follows the Bernoulli PDF and is dependent on the 146 FOR of DDOCR. In addition, MCS is used to generate the scenario for this uncertainty parameter.

147 In Eqs. (2) and (3), the amount of fault current flow through the relays depends on the value of network variables at the 148 moment before the fault. Generally, in most papers, network variables calculate according to the power flow problem, where the 149 capacity of distributed generations are considered constant ${ }^{8,19}$. However, it is possible that the variable DGs, such as the wind 150 turbine system are exist in the distribution network, so the level of its production capacity varies according to the weather 151 conditions at different times. Also in distribution network, DGs operate in optimal operation conditions, hence its supplied power 152 level varies at different times. Therefore, it is possible that employment of the results of the power flow calculations lead to error 153 in fault current calculation, so the obtained results of the SCOPC scheme will not be reliable. In this paper, the daily operation 
problem of distribution network is used to calculate the network variables, which will be another innovation of this paper. Finally,

155 the operation model of distribution network is formulated as follows:

$\min \quad O C=\sum_{s \in S C_{2}} \pi_{s} \sum_{h \in \Psi_{H}}\left(\lambda_{h, s} P_{r e f, h, s}^{S}+\sum_{g \in \Psi_{D G}} \beta_{g} P_{g, h, s}^{D G}\right)$

Subject to:

$$
\begin{aligned}
P_{n, h, s}^{S}+\sum_{g \in \Psi_{D G}} A_{n, g}^{G} P_{g, h, s}^{D G}-\sum_{j \in \Psi_{N}} A_{n, j}^{L} P_{n, j, h, s}^{L} & =P_{n, h, s}^{D} \forall n, h, s \\
Q_{n, h, s}^{S}+\sum_{g \in \Psi_{D G}} A_{n, g}^{G} Q_{g, h, s}^{D G}-\sum_{j \in \Psi_{N}} A_{n, j}^{L} Q_{n, j, h, s}^{L} & =Q_{n, h, s}^{D} \\
& \forall n, h, s
\end{aligned}
$$

$P_{n, j, h, s}^{L}=$

$G_{n, j}^{L}\left(V_{n, h, s}\right)^{2}-V_{n, h, s} V_{j, h, s}\left\{\begin{array}{l}G_{n, j}^{L} \cos \left(\delta_{n, h, s}-\delta_{j, h, s}\right) \\ +B_{n, j}^{L} \sin \left(\delta_{n, h, s}-\delta_{j, h, s}\right)\end{array}\right\}$

$$
\forall n, j, h, s
$$

$Q_{n, j, h, s}^{L}=$

$-B_{n, j}^{L}\left(V_{n, h, s}\right)^{2}+V_{n, h, s} V_{j, h, s}\left\{\begin{array}{l}B_{n, j}^{L} \cos \left(\delta_{n, h, s}-\delta_{j, h, s}\right) \\ -G_{n, j}^{L} \sin \left(\delta_{n, h, s}-\delta_{j, h, s}\right)\end{array}\right\}$

$$
\forall n, j, h, s
$$

$\sqrt{\left(P_{n, j, h, s}^{L}\right)^{2}+\left(Q_{n, j, h, s}^{L}\right)^{2}} \leq \bar{S}_{n, j}^{L} \quad \forall n, j, h, s$

$\sqrt{\left(P_{n, h, s}^{S}\right)^{2}+\left(Q_{n, h, s}^{S}\right)^{2}} \leq \bar{S}_{n}^{S} \quad \forall n, h, s$

$\underline{V} \leq V_{n, h, s} \leq \bar{V} \quad \forall n, h, s$

$0 \leq P_{g, h, s}^{D G} \leq \bar{P}_{g}^{D G} \quad \forall g \in \Psi_{D G} \backslash \Psi_{R E S}, h, s$

$\underline{Q}_{g}^{D G} \leq Q_{g, h, s}^{D G} \leq \bar{Q}_{g}^{D G} \quad \forall g \in \Psi_{D G} \backslash \Psi_{R E S}, h, s$

$0 \leq P_{g, h, s}^{D G}=\bar{P}_{g, h, s}^{D G} \quad \forall g \in \Psi_{R E S}, h, s$

156 The objective function of the optimal operation problem is presented in Eq. (13), which minimizes the expected total operation 157 cost of SDGs and the expected received energy cost from the upstream network ${ }^{19}$. Constraints (14)-(17) are correspond to the 158 power flow equations, which represent the active and reactive power balance in network buses, and active and reactive power 159 flowing through distribution lines, respectively ${ }^{18,20}$. In addition, the technical constraint of the distribution network including 160 line and substation capacity limits and bus voltage limitations are expressed in Eqs. (18)-(20), respectively ${ }^{21}$. Constraints (21) 161 and (22) represent the corresponding equations with the capability curve of the SDG, except for its renewable type such as wind turbine system, which its generation active and reactive powers limits are presented in Eqs. (21) and (22), respectively ${ }^{22,23}$. 
Finally, the corresponding constraint with the variable DG is presented in Eq. (23) ${ }^{23}$. According to the IEEE1547 standard ${ }^{24}$,

164 the variable DGs are generally used for energy production and their reactive power control capability is not considered, so following this concept, only the active power constraint of them is considered in this paper.

In Eqs. (13)-(23) of the problem, the load consumption, the energy price and production power of renewable SDG like a wind turbine system are as uncertainty. Therefore, in this paper, stochastic programming is used to model these parameters. In this method, MCS method is used to generate a high number of scenarios corresponding to normal/Weibull PDF for load consumption and energy price/production power of renewable SDG ${ }^{25}$. Then, the Kantorovich mechanism is used as a scenario reduction method to achieve a low number of scenarios with a high probability of occurrence ${ }^{25}$.

In this paper, it is assumed that a fault occurs in the distribution line, so to calculate the fault current flow through the DDOCRs corresponding to the fault $f$, the fault location is selected as a new bus that called $k$, firstly. Voltage of this node at a time before the fault occurrence is calculated by KVL law. Then, the calculation process of the fault current flow through the DDOCRs is the calculation of the bus voltage after the fault occurrence, which can be calculated by Eq. (24). In this equation, $Z$ is equal to the network matrix admittance with considering bus $k$, and $Z$ is equal to the impedance of the fault agent. By calculating the bus voltages, the current flow through the distribution line after the fault occurrence is calculated according to Ohm's law as in Eq. (25). Finally, to extract a reliable OPC and the worst-case scenario, which corresponds to the maximum current flow through the DDOCRs, must be considered. Therefore, in this paper, as in Eq. (26), the fault current flow through a relay is equal to the maximum fault current flow through the distribution line in which the relay is located considering all simulation hours and all scenarios.

$$
\begin{array}{lc}
V_{n, h, s, f}^{F}=V_{n, h, s}-\frac{Z_{n, k(f)}}{Z_{k(f), k(f)}+Z^{f}} V_{k(f), h, s} & \forall n, h, s, f \\
I_{n, j, h, s, f}^{F}=\left|\frac{V_{n, h, s, f}^{F}-V_{j, h, s, f}^{F}}{Z_{n, j}^{L}}\right| & \forall n, j, h, s, f \\
I_{r, f}^{S C}, I_{r, f, b}^{S C}=\max \left(I_{n, j, h, s, f}^{F}\right) & \forall r(n, j), f
\end{array}
$$

\section{3. Solution method}

182 The OPC problem will be complex one due to the employment of a number of high adjustment variables for dual setting 183 DDOCR, especially in larger distribution networks. Also, the optimal operation problem of distribution network has such 184 conditions. Therefore, it is predicted that the employment of an evolutionary algorithm will not be able to achieve a reliable 185 response. To overcome for this issue, this paper uses a combination of meta-heuristic algorithms such as combination of GWO $186{ }^{26}$ and TLBO ${ }^{27}$ to solve the proposed problems. It is noteworthy that in this case, the number of updating steps of the decision 187 variables value is more than employment an evolutionary algorithm, so it is possible to obtain an optimal solution considering 
low standard deviation with less computational complexity. Another advantage of this algorithm is the lack of adjustment 189 parameters in this algorithm ${ }^{26,27}$.

190 In the proposed solution method for SCOPC, to consider the constraint in Eq. (12), this term is added as a penalty function

191 like $\sum_{r, f, b, w} \mu_{r, f, b, w} \max \left(0, C T I-\left(t_{r, f, b, w}^{r v / b}-t_{r, f, w}^{f w / p}\right)\right)$ to the objective function in Eq. (1), which $\mu \geq 0$ represents the penalty variable

192 or Lagrange multiplier ${ }^{2}$. Therefore, to solve the new problem based on the Eqs. (27)-(29), the initial population is randomly 193 obtained for decision variables, i.e. $A^{f w / p}, A^{r v / b}, B^{f w / p}, B^{r v / b}, I P^{f w / p}, I P^{r v / b}, T D S^{f w / p}, T D S^{r v / b}$ and $\mu$ corresponding to constraints in Eqs.

194 (4)-(11) and (29), firstly. Then, the fitness function in Eq. (27) and operating time of DDOCRs in (2) and (3), is calculated for 195 the resulting population. The optimal point for the initial condition corresponds to the minimum point for the fitness function. In the next steps, the decision variables are executed. Therefore, first the updating steps of the TLBO algorithm, i.e. the teacher phase and then the student phase, are implemented. In the third phase, the updating process of GWO is presented. These steps continue until the convergence point is reached, in which it is assumed that the convergence point is available after maximum iteration with the abbreviation iter $_{\max }$.

$$
\begin{aligned}
\min F_{1} & =\sum_{w \in \Psi_{s C_{1}}} \pi_{w}\left(\sum_{f \in \Psi_{F}}\left(\sum_{r \in \Psi_{R}} t_{r, f, w}^{f w / p}+\sum_{b \in \Psi_{B}} t_{r, f, b, w}^{r v / b}\right)\right) \\
& +\sum_{r, f, b, w} \mu_{r, f, b, w} \max \left(0, C T I-\left(t_{r, f, b, w}^{r v / b}-t_{r, f, w}^{f w / p}\right)\right)
\end{aligned}
$$

Subject to:

Constraints (2)-(11)

$\mu \geq 0$

200 The solving process of optimal operation problem of distribution network is the same as the solving process of SCOPC 201 problem. Therefore, the technical constraints of the network, (18)-(20), are added as a penalty function to the objective function 202 in Eq. (13), which is a new problem that provided in Eqs. (30)-(32). First, a random initial population for the decision variables $203 P^{D G}, Q^{D G}$, and $\mu$ is calculated based on constraints in Eqs. (21)-(23) and (32). Then the value of network variables are calculated 204 with the AC power flow equations that given in Eqs (16)-(17). Also, these equation is solved based on the backward-forward power flow method ${ }^{28}$. Finally, the fitness function will be evaluated using the Eq. (30) for the whole population of decision variables. The minimum point is optimal response. In the following, the updating steps will be implemented as the SCOPC problem solving process until the convergence point is reached. Finally, the flowchart of proposed algorithm is shown in Fig. 1. 


$$
\begin{aligned}
& \min \quad F_{2}=\sum_{s \in S C_{2}} \pi_{s} \sum_{h \in \Psi_{H}}\left(\lambda_{h, s} P_{r e f, h, s}^{S}+\sum_{g \in \Psi_{D G}} \beta_{g} P_{g, h, s}^{D G}\right)+ \\
& \sum_{n, h, s} \bar{\mu}_{n, h, s}^{v} \max \left(0, V_{n, h, s}-\bar{V}\right)+\underline{\mu}_{n, h, s}^{v} \max \left(0, \underline{V}-V_{n, h, s}\right) \\
& +\sum_{n, h, s} \bar{\mu}_{n, h, s}^{S} \max \left(0, \sqrt{\left(P_{n, h, s}^{S}\right)^{2}+\left(Q_{n, h, s}^{S}\right)^{2}}-\bar{S}_{n}^{S}\right)+ \\
& \sum_{n, h, s} \bar{\mu}_{n, j, h, s}^{L} \max \left(0, \sqrt{\left(P_{n, j, h, s}^{L}\right)^{2}+\left(Q_{n, j, h, s}^{L}\right)^{2}}-\bar{S}_{n, j}^{L}\right)
\end{aligned}
$$

\section{Subject to:}

Constraints (14)-(23)

$\mu \geq 0$ 


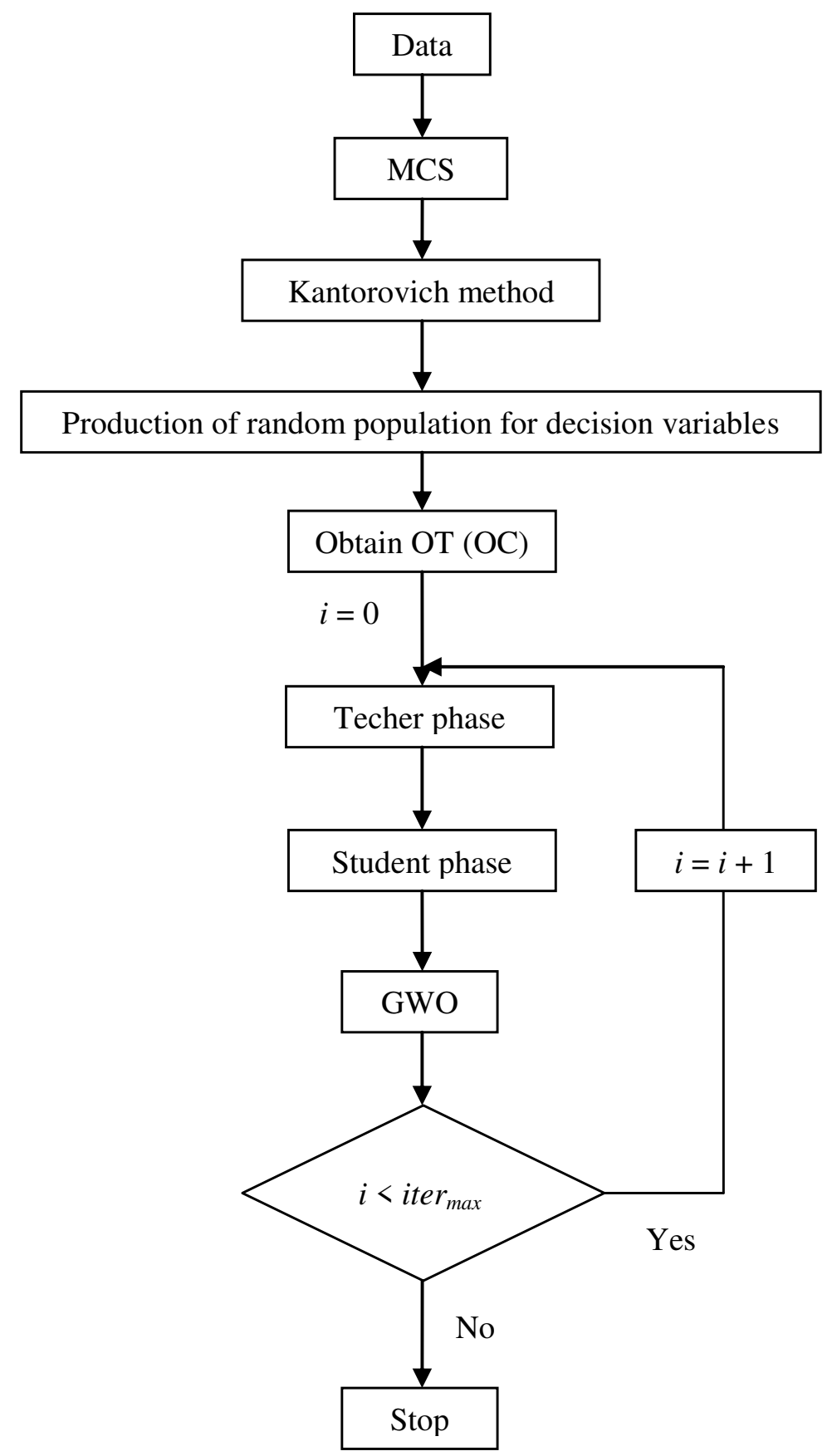

Fig. 1 Flowchart of proposed solution algorithm

\section{4. Numerical results}

212 In this section, the proposed problem is simulated on a standard 33-bus and 119-bus distribution networks ${ }^{29}$ in the MATLAB

213 software environment. Note that the MCS method in the SCOPC scheme generates 30 scenarios for the uncertainty of the

214 DDOCRs unavailability, which is assumed the FOR of relays is equal to $0.5 \%$. But it produces 1000 scenarios for the uncertainty

215 of load consumption, energy prices, and production power of renewable SDGs, assuming a standard deviation of $10 \%$ in the

216 distribution network operation problem. Then, in this problem, the Kantorovich method selects 30 scenarios from the generated 217 scenarios, which have a high probability of occurrence. In addition, for the proposed combined GWO and TLBO algorithm, the 
population and the maximum of coverage iteration are selected equal to 50 and 3000, respectively.

\subsection{Case Studies}

A) IEEE 33-bus radial distribution system: Single Line diagram of IEEE 33-bus radial distribution system with $12.66 \mathrm{kV}$ base voltage and 1 MVA base power is plotted in Fig. $2{ }^{29}$. The characteristic of lines and distribution substation are provided in ${ }^{29}$. The allowable voltage limits is equal [0.9 p.u, 1.05 p.u.]. Also, the load profile and the daily energy price curve are presented in

${ }^{30}$. It is noteworthy that in terms of network load, time interval 1:00 to 7:00 is equal to the low load case, which has an energy price of $16 \$ / M W h$. Also, the time interval 17:00 to 22:00 is correspond to the peak load case which the energy price is 30\$/MWh. Other time intervals are correspond to middle load case and its energy price is $24 \$ / \mathrm{MWh}$. The network also has three nonrenewable SDGs with a capacity of $500 \mathrm{kVA}$ and a fuel price of $20 \$ / \mathrm{MWh}$ on the busses 14, 27 and 32, whose them DDOCRs are marked with numbers 35, 37 and 38, respectively. Two renewable SDGs (wind turbine) with $400 \mathrm{~kW}$ capacity are installed in busses 6 and 20, which DDOCRs 34 and 36 are located in these buses, respectively. The daily wind power rate curve is also presented in ${ }^{30}$. In addition, the left/hand relays of bus $n$ are considered as $\mathrm{Rn} / \mathrm{Rn}$ '. It is assumed that the fault location is in the center of the distribution lines, which its details are presented in Table 1. In this table, the operation modes of different DDOCRs for different fault locations are provided. Based on ${ }^{2}$, relays in both sides (left and right) of each fault location act as the primary mode. An example of how to determine the backup relays is given. It is assumed that a fault has occurred in the distribution line between buses 13 and 14. In this case, R13' and R14 in direction of fw/p are in the primary protection mode. For the R13' in direction fw/p, the R13 in direction rv/b are in the backup protection mode and for the R14 in direction fw/p, the R14' and R35 in direction $\mathrm{rv} / \mathrm{b}$ will be in the backup mode.

In the SCOPC scheme, the minimum and maximum values for A is equal to 0.14 and 13.5 , respectively. These values for B are equal to 0.02 and 1, respectively. For TDS, they are equal to 0.1 and $1 \mathrm{sec}$. The CTI is considered equal to $0.3 \mathrm{sec}$. The minimum and maximum values of PC for a relay (in the distribution line or in the SDG path) are equal to $100 \%$ and $130 \%$ current of the distribution line or SDG in the pre-fault occurrence, respectively.

B) IEEE 119-bus radial distribution system: The single line diagram of the IEEE 119-bus radius distribution network is shown in Fig. 3, which its distribution lines and load peak characteristics are provided in ${ }^{29}$. The daily load factor curve is also expressed in ${ }^{30}$. This network also has $400 \mathrm{kVA}$ non-renewable SDGs with 20 \$/MWh fuel price that installed on busses 24, 39, 48, 73, 94 and 109, whose them DDOCRs are marked with numbers 120-125, respectively. Also, the $400 \mathrm{~kW}$ renewable SDGs with 126 to 129 relays are connected to buses 4, 30, 65 and 101. As the IEEE 33-bus distribution network, it is assumed that at the center of each distribution line there is a possibility of fault occurrence. The process of determining the primary and backup protection mode for relays appropriate to different fault locations such as Table 1. Finally, other problem data such as energy prices, the allowable limits of DDOCR and CTI adjustment parameters are the same as the IEEE 33-bus distribution network. 


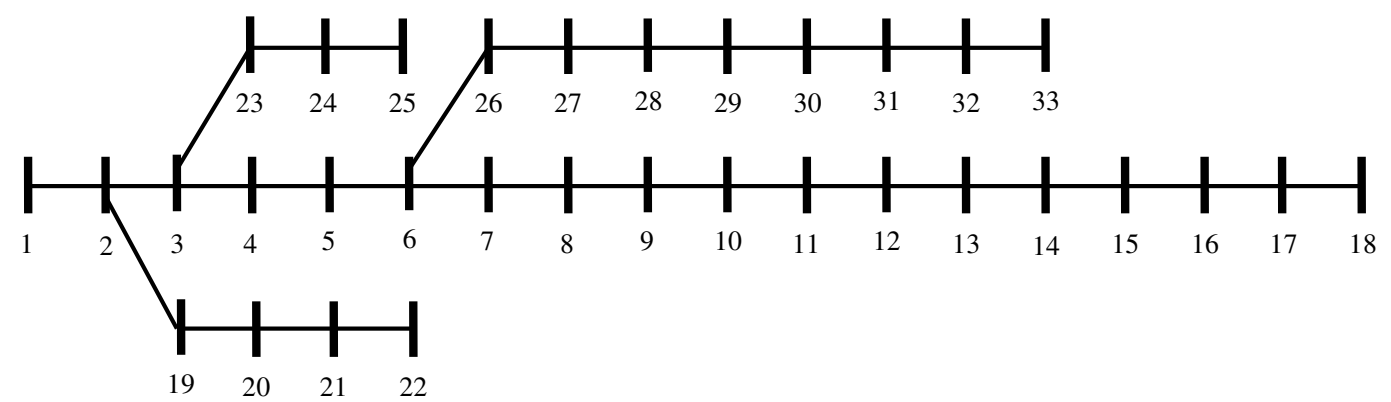

Fig. 2 Single line diagram of IEEE 33-buses distribution network ${ }^{29}$

Table 1: Operation modes of DDOCRs for different fault location

\begin{tabular}{|c|c|c|c|c|c|}
\hline Fault & Fault location (between busses) & $\mathrm{P}_{1}$ & $\mathrm{~B}$ & $\mathrm{P}_{2}$ & $\mathrm{~B}$ \\
\hline 34 & $1-2$ & $\mathrm{R} 1$ 'fw/p & R1rv/b & $\mathrm{R} 2 \mathrm{fw} / \mathrm{p}$ & R2'rv/b, R2"rv/b \\
\hline 35 & $2-3$ & $\mathrm{R} 2$ 'fw/p & $\mathrm{R} 2 \mathrm{rv} / \mathrm{b}$ & R3fw/p & R3'rv/b, R3"rv/b \\
\hline 36 & $3-4$ & R3'fw/p & $\mathrm{R} 3 \mathrm{rv} / \mathrm{b}$ & $\mathrm{R} 4 \mathrm{fw} / \mathrm{p}$ & R4'rv/b \\
\hline 37 & $4-5$ & R4'fw/p & $\mathrm{R} 4 \mathrm{rv} / \mathrm{b}$ & $\mathrm{R} 5 \mathrm{fw} / \mathrm{p}$ & R5'rv/b \\
\hline 38 & $5-6$ & $\mathrm{R} 5$ ' $\mathrm{fw} / \mathrm{p}$ & R5rv/b & R6fw/p & R6'rv/b, R6"rv/b, R34rv/b \\
\hline 39 & $6-7$ & R6'fw/p & R6rv/b, R34rv/b & R7fw/p & R7'rv/b \\
\hline 40 & $7-8$ & R7'fw/p & $\mathrm{R} 7 \mathrm{rv} / \mathrm{b}$ & R8fw/p & R8'rv/b \\
\hline 41 & $8-9$ & R8'fw/p & R8rv/b & R9fw/p & R9'rv/b \\
\hline 42 & $9-10$ & R9'fw/p & R9rv/b & R10fw/p & R10'rv/b \\
\hline 43 & $10-11$ & $\mathrm{R} 10$ 'fw/p & R10rv/b & R11fw/p & R11'rv/b \\
\hline 44 & $11-12$ & R11'fw/p & $\mathrm{R} 11 \mathrm{rv} / \mathrm{b}$ & R12fw/p & R12'rv/b \\
\hline 45 & $12-13$ & R12'fw/p & $\mathrm{R} 12 \mathrm{rv} / \mathrm{b}$ & R13fw/p & R13'rv/b \\
\hline 46 & $13-14$ & R13'fw/p & $\mathrm{R} 13 \mathrm{rv} / \mathrm{b}$ & R14fw/p & R14'rv/b, R35rv/b \\
\hline 47 & $14-15$ & R14'fw/p & R14rv/b, R35rv/b & $\mathrm{R} 15 \mathrm{fw} / \mathrm{p}$ & R15'rv/b \\
\hline 48 & $15-16$ & R15'fw/p & $\mathrm{R} 15 \mathrm{rv} / \mathrm{b}$ & R16fw/p & R16'rv/b \\
\hline 49 & $16-17$ & R16'fw/p & $\mathrm{R} 16 \mathrm{rv} / \mathrm{b}$ & R17fw/p & R17'rv/b \\
\hline 50 & $17-18$ & R17'fw/p & $\mathrm{R} 17 \mathrm{rv} / \mathrm{b}$ & R18fw/p & - \\
\hline 51 & $2-19$ & R2"fw/p & $\mathrm{R} 2 \mathrm{rv} / \mathrm{b}$ & R19fw/p & R19'rv/b \\
\hline 52 & $19-20$ & R19'fw/p & R19rv/b & R20fw/p & R20'rv/b, R36rv/b \\
\hline 53 & $20-21$ & R20'fw/p & R20rv/b, R36rv/b & R21fw/p & R21'rv/b \\
\hline 54 & $21-22$ & R21'fw/p & $\mathrm{R} 21 \mathrm{rv} / \mathrm{b}$ & R22fw/p & - \\
\hline 55 & $3-23$ & R3"fw/p & R3rv/b & R23fw/p & R23'rv/b \\
\hline 56 & $23-24$ & R23'fw/p & $\mathrm{R} 23 \mathrm{rv} / \mathrm{b}$ & R24fw/p & R24'rv/b \\
\hline 57 & $24-25$ & R24'fw/p & $\mathrm{R} 24 \mathrm{rv} / \mathrm{b}$ & $\mathrm{R} 25 \mathrm{fw} / \mathrm{p}$ & - \\
\hline 58 & $6-26$ & R6"fw/p & R6rv/b & R26fw/p & R26'rv/b \\
\hline 59 & $26-27$ & R26'fw/p & R26rv/b & R27fw/p & R27'rv/b, R37rv/b \\
\hline 60 & $27-28$ & R27'fw/p & R27rv/b, R37rv/b & R28fw/p & R28'rv/b \\
\hline 61 & $28-29$ & R28'fw/p & $\mathrm{R} 28 \mathrm{rv} / \mathrm{b}$ & R29fw/p & R29'rv/b \\
\hline 62 & $29-30$ & R29'fw/p & R29rv/b & R30fw/p & R30'rv/b \\
\hline 63 & $30-31$ & R30'fw/p & R30rv/b & R31fw/p & R31'rv/b \\
\hline 64 & $31-32$ & R31'fw/p & $\mathrm{R} 31 \mathrm{rv} / \mathrm{b}$ & R32fw/p & R32'rv/b, R38rv/b \\
\hline 65 & $32-33$ & R32'fw/p & R32rv/b, R38rv/b & R33fw/p & - \\
\hline
\end{tabular}

R2": DDOCR in left side of line 2-19, R3": DDOCR in left side of line 3-23, R6"= DDOCR in left side of line 6-25 


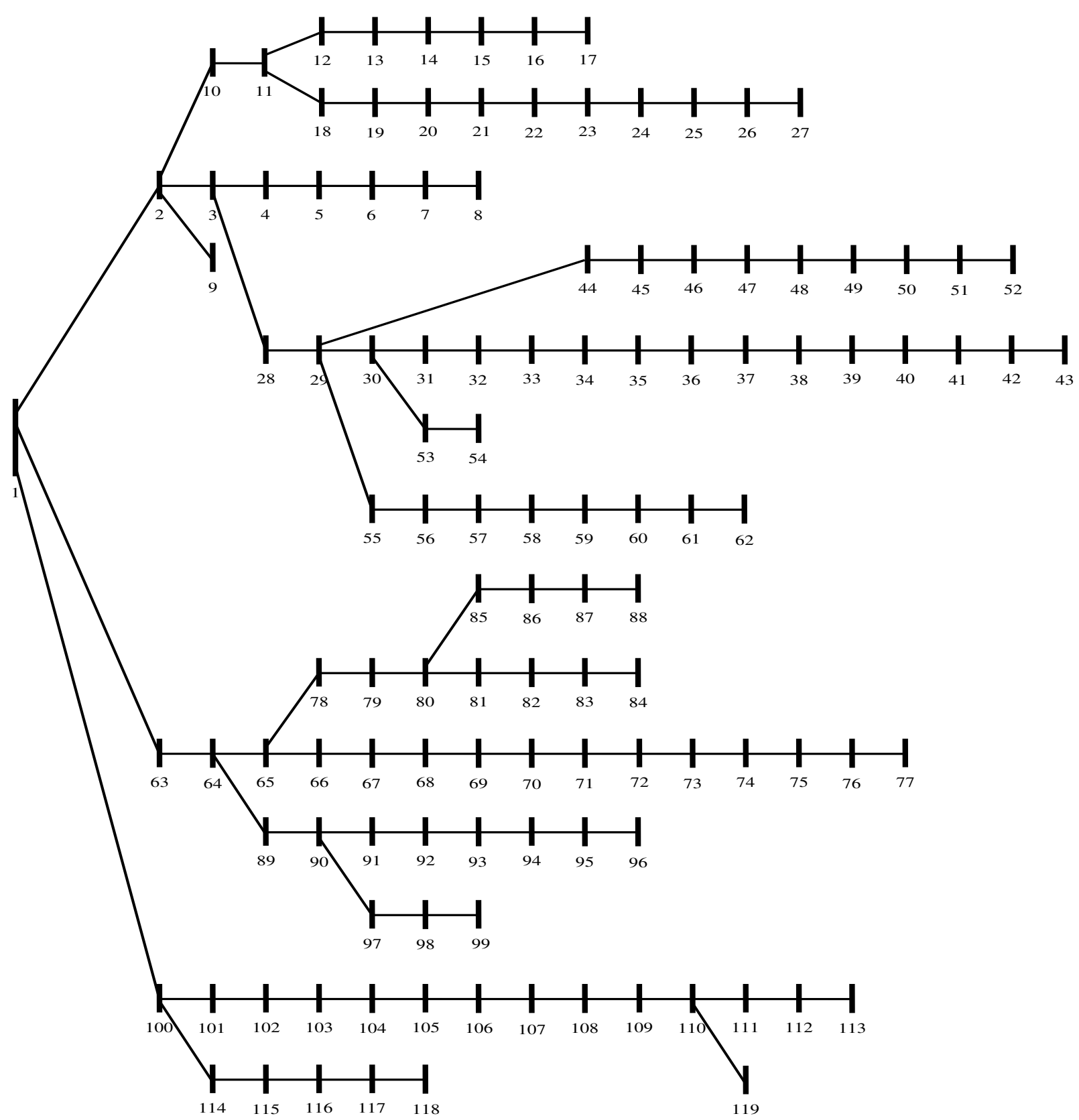

Fig. 3 Single line diagram of IEEE 119-buses distribution network ${ }^{29}$

\section{$4.2 \quad$ Results}

In this section, the capabilities of the proposed scheme on the IEEE 33-bus and 119-bus distribution networks are evaluated, which the numerical results obtained by coding the proposed problem in MATLAB software.

A) Investigating the potential of the proposed solution algorithm: The statistical results of the obtained optimal response from different solvers such as sine cosine algorithm (SCA) ${ }^{12}$, krill herd optimization (KHO) algorithm ${ }^{31}, \mathrm{GWO}^{26}$, TLBO ${ }^{27}$ and combination of GWO and TLBO are provided in Table 2. It is noteworthy that for all proposed algorithms, the population size is 50 and the maximum of convergence iteration is 3000 , and regulation parameters for SCA and KHO solver are presented in ${ }^{12}$ and ${ }^{31}$, respectively. Also, to achieve the statistical results such as the mean of the final optimal response and its standard deviation, each proposed algorithm is repeated 20 times. Based on the results that presented in Table 2, it can be seen that the 
proposed algorithm (combination of GWO and TLBO) for the objective functions in Eqs. (1) and (13) based on 33-bus network data has been able to coverage at the 731 and 1273 iterations with 12.7 and 82.1 computational time, respectively. These values for the 119-bus network are at the 1046 and 1836 iterations with 21.2 and 103.5 computational times, respectively. Comparing these results with the obtained convergence iteration and computational time for other proposed algorithms indicates that the combined algorithm GWO and TLBO can be reached to minimum value of objective function (OT and OC) in the shortest time and lowest iteration compared to SCA, KHO, GWO and TLBO. In addition, the obtained mean value of OT (OC) with combination of GWO and TLBO are equal to $52.32 \mathrm{sec}(902.73$ \$) and $123.62 \mathrm{sec}(2853.15$ \$) in the SCOPC (network optimal operation) scheme of the 33-bus and 119-bus distribution networks, respectively, which in comparison to other algorithms that described in this section are the lowest. This also indicates the ability of the proposed algorithm to achieve the lowest optimal point compared to other algorithms. Finally, based on rows of 7 and 16 in Table 2, it can be seen that the standard deviation of the objective functions of OT and OC for the combination of GWO and TLBO algorithm is much lower than for other algorithms.

Also, the standard deviation values of these objective functions in the two 33-bus and 119-bus distribution networks are very close to each other, but this is not established in the other algorithms that presented in Table 2. Hence, it can be said that the proposed algorithm is able to achieve the best solution including low standard deviation, which is very little dependent on the problem data. But in SCA, KHO, GWO and TLBO solvers, increasing the scale of problem data (comparing the results of the 119-bus network to the 33-bus network) increases the standard deviation of the optimal point, so it is predicted that in large real networks, these algorithms don't have the ability to achieve the reliable optimal solution. However, since the combination of GWO and TLBO has the same and low standard deviation values in the two mentioned networks, the consistency of this algorithm is slightly dependent on the data scale of the problem and is suitable for large scale networks. It is noteworthy that all the benefits provided in this section confirm the fourth innovation in Section 1.

Table 2: Statistical analysis results of different algorithms

\begin{tabular}{|c|c|c|c|c|c|c|c|c|c|c|}
\hline \multicolumn{11}{|c|}{ 33-bus distribution network } \\
\hline Problem & \multicolumn{5}{|c|}{ SCOPC } & \multicolumn{5}{|c|}{ Distribution network operation } \\
\hline Algorithm & SCA & KHO & GWO & TLBO & GWO+TLBO* & SCA & KHO & GWO & TLBO & GWO+TLBO* \\
\hline Convergence iteration & 1294 & 1472 & 956 & 937 & 731 & 1783 & 1836 & 1503 & 1421 & 1273 \\
\hline $\begin{array}{l}\text { Calculation time }(\mathrm{s}) \text { at } \\
\text { convergence iteration }\end{array}$ & 29.5 & 32.2 & 18.1 & 18.6 & 12.7 & 127.4 & 136.8 & 94.1 & 93.2 & 82.1 \\
\hline $\begin{array}{c}\text { Mean value of } \\
\text { objective function }\end{array}$ & 66.35 & 64.94 & 58.21 & 56.33 & $52.32(\mathrm{~s})$ & 978.67 & 971.11 & 953.27 & 945.16 & $902.73(\$)$ \\
\hline $\begin{array}{l}\text { Standard deviation }(\%) \\
\text { for objective function }\end{array}$ & 4.04 & 3.59 & 2.12 & 1.92 & 0.97 & 4.24 & 4.18 & 2.57 & 2.31 & 1.02 \\
\hline \multicolumn{11}{|c|}{ 119-bus distribution network } \\
\hline Problem & \multicolumn{5}{|c|}{ SCOPC } & \multicolumn{5}{|c|}{ Distribution network operation } \\
\hline Algorithm & $\mathrm{SCA}$ & KHO & GWO & TLBO & $\mathrm{GWO}+\mathrm{TLBO}^{*}$ & SCA & KHO & GWO & TLBO & GWO+TLBO* \\
\hline Convergence iteration & 1711 & 1906 & 1409 & 1312 & 1046 & 2577 & 2737 & 2208 & 2111 & 1836 \\
\hline $\begin{array}{l}\text { Calculation time (s) at } \\
\text { convergence iteration }\end{array}$ & 51.7 & 60.22 & 32.43 & 29.8 & 21.2 & 194.72 & 241.44 & 150.61 & 147.27 & 103.5 \\
\hline $\begin{array}{c}\text { Mean value of } \\
\text { objective function }\end{array}$ & 171.81 & 163.55 & 145.37 & 141.23 & $123.62(\mathrm{~s})$ & 3583.26 & 3485.19 & 3121.59 & 3064.23 & $2853.15(\$)$ \\
\hline $\begin{array}{c}\text { Standard deviation for } \\
\text { objective function }\end{array}$ & 5.89 & 5.34 & 3.87 & 3.12 & 0.99 & 6.94 & 6.11 & 4.22 & 3.99 & 1.05 \\
\hline
\end{tabular}


distribution networks in the presence of non-renewable and renewable SDGs (wind system type) are provided. Fig. 4 shows the expected daily curve of SDGs active power. Based on Fig. 4(a), it can be seen that the active power of the wind turbine system has different values for different hours, which is due to changes in wind speed during the day and night. Also, according to Fig. 4(b), the non-renewable SDGs in the two mentioned networks are off for 1:00 to 7: 00 and don't inject the active power into the network. Because based on the data in section 4-1, at these hours the purchased energy price from the upstream network is equal to $16 \$ / \mathrm{MWh}$, while the fuel price of non-renewable SDGs is equal to $20 \$ / \mathrm{MWh}$. Therefore, in order to minimize the expected operation cost (OC), the most optimal status is for consumers to be fed by the upstream network. But in other hours, the opposite is true, so these SDGs inject their maximum active power into the network. In addition, Table 3 presented the advantages of the proposed optimal operation in proportion to the optimal scheduling for SDGs as illustrated in Fig. 4. Based on this, it can be seen that the operation cost of the IEEE 33-bus distribution network, regardless of the presence of SDGs is equal to 1950 \$, the energy losses equal to 1.878 p.u., and the maximum voltage drop equal to 0.087 p.u., However, these operating indices, if SDGs are present are reduced to $902.73 \$, 0.8988$ p.u and 0.0437 p.u., respectively, but the maximum overvoltage is created to 0.0115 p.u., which is much less than its allowable limit i.e. 0.05 p.u. (1.05-1). Therefore, these conditions indicate the improvement of the mentioned indicators by about $53.7 \%$ ((1950-902.73)/1950), 51.1\% ((1.878-0.8988)/1.878) and 49.8\% ((0.087-0.0437)/0.087), respectively. This situation is also established for IEEE 119-buses distribution network, so that the expected operation costs, energy losses and maximum voltage drop in the presence of SDGs in the network compared to the mentioned distribution network without the presence of SDGs are reduced by about $38 \%, 47.8 \%$ and $43.2 \%$, respectively.

As said in Section 1, researches generally uses the power flow analysis results of distribution network with considering constant injection active power of SDGs to calculate the fault current flow through the relays. The OPC (it is not consider security situation) scheme is considered appropriate under case I. In other words, it is assumed that the injected active power of all SDGs is equal to their maximum capacity, and other data is proportional to the peak hours. The OPC scheme is also evaluated based on the results of the optimal operation of the distribution network in the case II. The results of this section are presented in Table

311 4. According to this table, the total operation time of all DDOCRs in the two primary and backup protection modes considering

312 all the fault locations that provided in Table 1 for case I in the two 33-bus and 119-bus networks are 55.83 sec and 135.95 sec,

313 respectively. These total operation times for case II are $49.12 \mathrm{sec}$ and $120.41 \mathrm{sec}$, respectively. Therefore, the computational

314 difference between the methods that presented in cases I and II for the two networks is about $13 \%$. However, since the optimal 315 operation scheme considers the voltage changes of different buses in proportion to the changes in the power level of SDGs and 316 loads, so case II achieves more accurate results for calculating the fault current. From this, it can be said that the OPC results 317 obtained in the case II are more reliable than the case I, which confirms the third innovation in Section 1. 


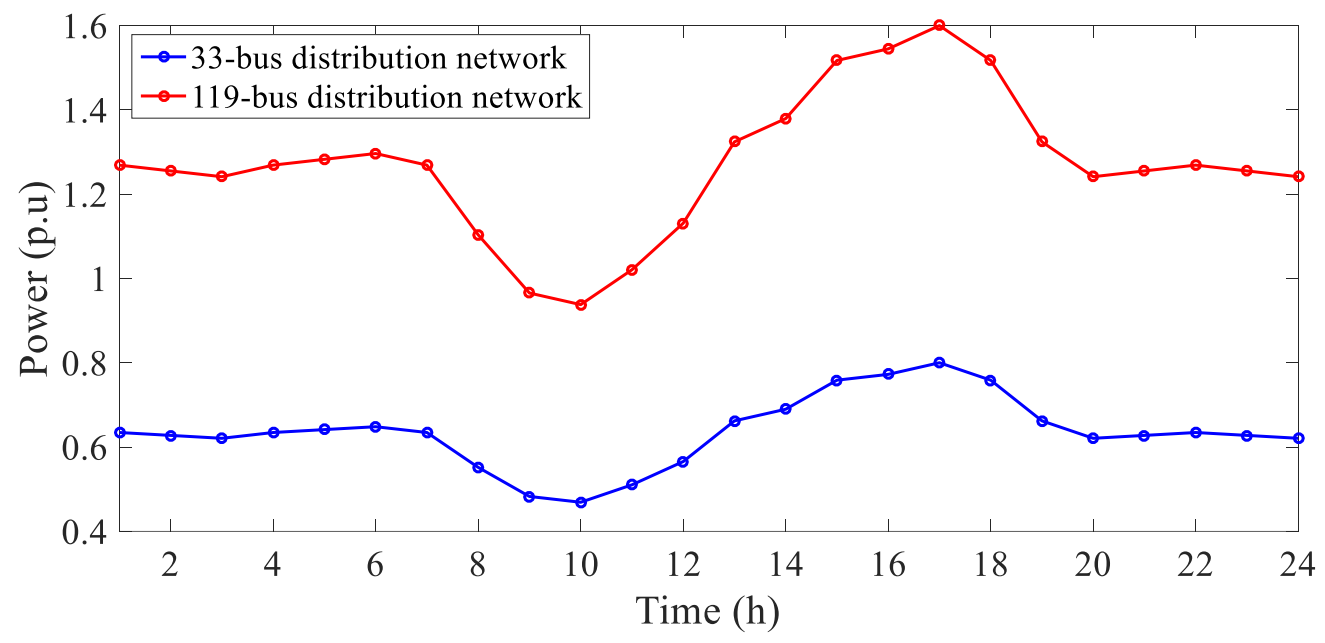

(a)

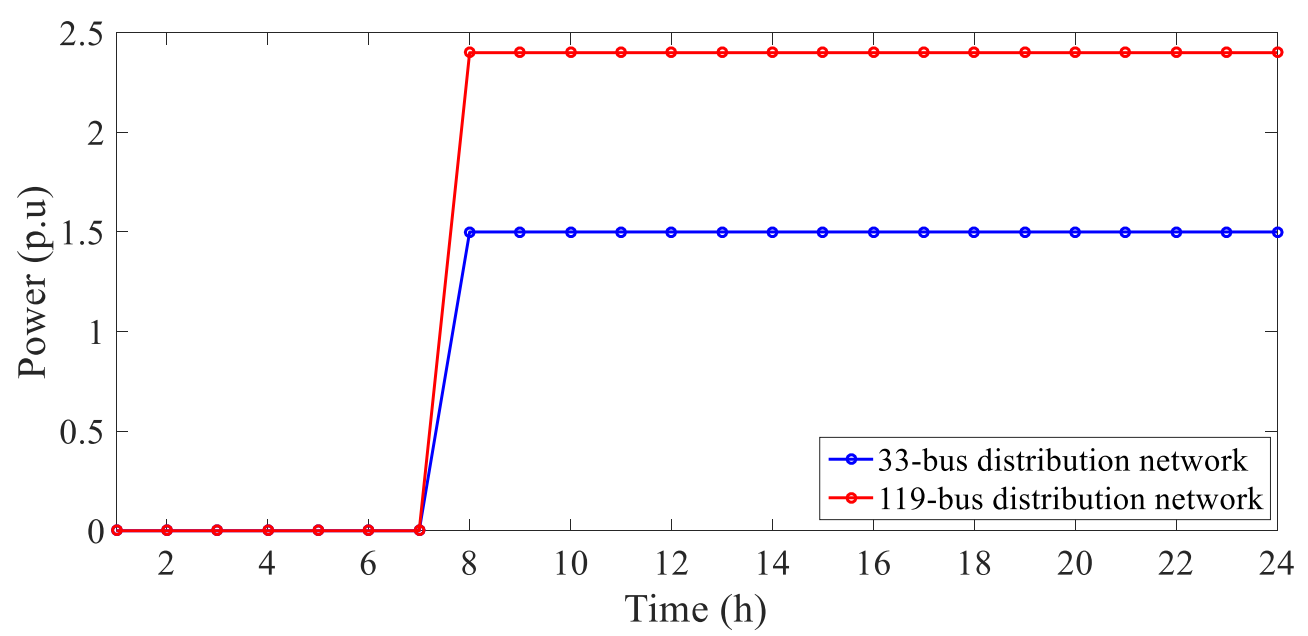

(b)

Fig. 4 Expected active power daily curve, a) renewable SDGs, b) non-renewable SDGs

Table 3: Expected operation indices values

\begin{tabular}{cccc}
\hline \multicolumn{5}{c}{ 33-bus distribution network } \\
\hline Case & Operation cost $(\$)$ & Energy loss (p.u) & Maximum value of overvoltage/voltage drop (p.u) \\
\hline Power flow analysis & 1950 & 1.878 & $0 / 0.087$ \\
\hline Optimal operation & 902.73 & 0.8988 & $0.0115 / 0.0437$ \\
\hline \multicolumn{4}{c}{ 119-bus distribution network } \\
\hline Case & Operation cost $(\$)$ & Energy loss (p.u) & Maximum value of overvoltage/voltage drop (p.u) \\
\hline Power flow analysis & 4525.5 & 3.672 & $0 / 0.092$ \\
\hline Optimal operation & 2853.15 & 1.917 & $0.0143 / 0.0523$ \\
\hline
\end{tabular}

Table 4: OPC results based on optimal operation and power flow of distribution system in presence of SDGs

\begin{tabular}{cccc}
\hline Network & Case & Total operation time of all DDOCR (s) & Deviation (\%) \\
\hline \multirow{2}{*}{ 33-bus } & I & 55.83 & $(55.83-49.12) / 49.12=13.66 \%$ \\
\cline { 2 - 3 } & II & 49.12 & \\
\hline 119-bus & I & 135.95 & $(129.95-120.41) / 120.41=12.9 \%$ \\
\cline { 2 - 3 } & II & 120.41 & \\
\hline
\end{tabular}


C) Proposed SCOPC scheme capabilities: In this section, five case studies are presented to evaluate the proposed SCOPC 324 scheme capabilities. The details of them are as follows:

- Case I: Investigation of the OPC for overcurrent relays (OCRs) considering the constant value of the pickup current, which is provided in traditional OPCs. So that the OCR pickup current is generally equal to $120 \%$ of the current flow through the OCR before the fault occurrence, and the values of A and B are equal to 0.14 and 0.02 , respectively.

- Case II: investigation of the OPC for OCRs that the values of A and B are equal to 0.14 and 0.02 .

- Case III: investigation of the OPC for DOCRs that the values of A and B are equal to 0.14 and 0.02.

- Case IV: investigation of the OPC for DDCORs

- Case V: investigation of the SCOPC for DDCORs

The results of this section are shown in Table 5 for the two 33-bus and 119-bus distribution networks. As can be seen from this table, the OPC scheme for DDOCR in case IV with 8 parameters, i.e. $A^{f w / p}, A^{r v / b}, B^{f w / p}, B^{r v / b}, I P^{f w / p}, I P^{r v / b}, T D S^{f w / p}$ and $T D S^{r v / b}$, is able to achieve faster protection coordination than other case studies, which confirms the second innovation in Section 1 . It is noteworthy that this advantage is obtained in such a way that $A^{f w / p}$ and $B^{f w / p}$ are set to values greater than 0.14 and 0.02 , and $A^{r / / b}$ and $B^{r v / b}$ are around values of 0.14 and $0.02 . T D S^{f w / p}$ is set to its smallest value i.e. 0.1 second, but $T D S^{r v / b}$ is set to a higher value than $T D S^{r v / b}$ in cases I to III. In the following, the pickup current of DDOCRs in the case IV is less than that of the pickup current in cases I to III. Finally, the OT value for the SCOPC scheme in the case V for the two 33-bus and 119-bus networks are 52.32 $\mathrm{sec}$ and $123.62 \mathrm{sec}$, respectively. This fitness function are equal to $49.12 \mathrm{sec}$ and $120.61 \mathrm{sec}$ for the case IV, respectively. This time difference is due to the uncertainty of the unavailability of DDOCRs, which has a more reliable response confirming the first innovation capability in section 1.

Table 5: Results of different OPC or SCOPC schemes

\begin{tabular}{|c|c|c|c|c|c|c|c|c|c|c|}
\hline \multirow[t]{2}{*}{ Network } & \multirow[t]{2}{*}{ Case } & \multirow[t]{2}{*}{ OT (sec) } & \multicolumn{8}{|c|}{ Mean value of } \\
\hline & & & $A^{f w / p}$ & $A^{r v / b}$ & $B^{f w / p}$ & $B^{r v / b}$ & $T D S^{f w / p}$ & $T D S^{r v / b}$ & $I P^{f w / p}$ & $I P^{r v / b}$ \\
\hline \multirow[t]{5}{*}{ 33-bus } & I & $\begin{array}{ll}63.38 \\
\end{array}$ & 0.14 & 0.14 & 0.02 & 0.02 & 0.1824 & 0.1824 & 0.8643 & 0.8643 \\
\hline & II & 59.84 & 0.14 & 0.14 & 0.02 & 0.02 & 0.1781 & 0.1781 & 0.8229 & 0.8229 \\
\hline & III & 53.63 & 0.14 & 0.14 & 0.02 & 0.02 & 0.1000 & 0.2361 & 0.8177 & 0.8175 \\
\hline & IV & 49.12 & 1.1263 & 0.1463 & 0.3317 & 0.0291 & 0.1000 & 0.2521 & 0.8038 & 0.8035 \\
\hline & V & 52.32 & 1.1271 & 0.1468 & 0.3345 & 0.0299 & 0.1000 & 0.2578 & 0.8072 & 0.8070 \\
\hline \multirow[t]{5}{*}{ 119-bus } & I & 138.64 & 0.14 & 0.14 & 0.02 & 0.02 & 0.1611 & 0.1611 & 0.7245 & 0.7245 \\
\hline & II & 133.49 & 0.14 & 0.14 & 0.02 & 0.02 & 0.1592 & 0.1592 & 0.6922 & 0.6922 \\
\hline & III & 125.11 & 0.14 & 0.14 & 0.02 & 0.02 & 0.1000 & 0.2018 & 0.6891 & 0.6889 \\
\hline & IV & 120.41 & 1.1936 & 0.1458 & 0.3119 & 0.0284 & 0.1000 & 0.2374 & 0.6785 & 0.6783 \\
\hline & $\mathrm{V}$ & 123.62 & 1.1971 & 0.1465 & 0.3134 & 0.0293 & 0.1000 & 0.2403 & 0.6791 & 0.6788 \\
\hline
\end{tabular}

\section{Conclusions}

In this paper, SCOPC scheme for coordination protection of dual setting DDOCRs in distribution network with renewable and non-renewable SDGs is presented. The SCOPC scheme minimizes the expected total operation time of these relays in the two primary and backup protection modes considering several fault locations, which is constrained to the allowable limits of DDOCR adjustment parameters and CTI limitations. In this problem, the unavailability of DDOCRs is uncertain, and stochastic 
programming is used to model it. In addition, this paper calculates the fault current flow through the relay corresponding to the stochastic operation results of the distribution network, which minimizes the expected total operation cost of network and nonrenewable SDGs, while constrained the equations of the optimal AC power flow in the distribution network. Also in this problem, stochastic programming is applied to model the uncertainties of load consumption, energy price and production power of renewable SDGs. Finally, the optimal solution for both optimal operation and SCOPC problems is achieved by a combined GWO and TLBO algorithms. Based on the obtained numerical results in this paper, it was seen that the proposed solver is able to obtain the most optimal point in lower coverage iteration and computational time with low standard deviation in responding to other SCA, KHO, GWO and TLBO algorithms. Also, the standard deviation of this algorithm has a low dependence on the data scale of the problem, so that for two networks and two problems (SCOPC and optimal operation) is about $1 \%$. In addition, based on the simulation results, it was illustrated that the SCOPC scheme based on optimal operation results is about 13\% more accurate than the SCOPC based on the power flow analysis considering the constant value for the active power of SDGs. Finally, the protection coordination of DDOCRs has a faster operation time due to the flexibility in different adjustment parameters than OCRs and DOCRs. Also, due to considering the unavailability of DDOCRs in the SCOPC scheme, the obtained results in this scheme have better reliability and confidence than the OPC scheme.

\section{Acknowledgements}

This research was funded by the Universiti Teknologi Malaysia (Post-Doctoral Fellowship Scheme grant 05E09, RUG grants 4B482, 01M44, 02M18, 05G88).

\section{Author Contributions}

All authors participated in the discussion and wrote the manuscript.

\section{References}

374 1. Xu, L., Chow, M.-Y., Timmis, J. \& Taylor, L. S. Power distribution outage cause identification with imbalanced data using artificial immune recognition system (AIRS) algorithm. IEEE Trans. Power Syst. 22, 198-204 (2007).

2. Najy, W. K. A., Zeineldin, H. H. \& Woon, W. L. Optimal protection coordination for microgrids with grid-connected and islanded capability. IEEE Trans. Ind. Electron. 60, 1668-1677 (2012).

3. Zeineldin, H. H., Sharaf, H. M., Ibrahim, D. K. \& Abou El-Zahab, E. E.-D. Optimal protection coordination for meshed distribution systems with DG using dual setting directional over-current relays. IEEE Trans. Smart Grid 6, $115-123$ (2014). 
4. Ghobadpour, S., Gandomkar, M. \& Nikoukar, J. Determining Optimal Size of Superconducting Fault Current Limiters to Achieve Protection Coordination of Fuse-Recloser in Radial Distribution Networks with Synchronous DGs. Electr. Power Syst. Res. 185, 106357 (2020).

5. Xu, L., Wang, C. \& Li, H. Decision and coordination of low-carbon supply chain considering technological spillover and environmental awareness. Sci. Rep. 7, 1-14 (2017).

6. Khurshaid, T. et al. Modified particle swarm optimizer as optimization of time dial settings for coordination of directional overcurrent relay. J. Electr. Eng. Technol. 14, 55-68 (2019).

7. Sulaiman, M., Ahmad, A., Khan, A. \& Muhammad, S. Hybridized symbiotic organism search algorithm for the optimal operation of directional overcurrent relays. Complexity 2018, (2018).

8. Sorrentino, E. \& Rodríguez, J. V. A novel and simpler way to include transient configurations in optimal coordination of directional overcurrent protections. Electr. Power Syst. Res. 180, 106127 (2020).

9. Elmitwally, A., Kandil, M. S., Gouda, E. \& Amer, A. Mitigation of DGs impact on variable-topology meshed network

10. Farzinfar, M. \& Jazaeri, M. A novel methodology in optimal setting of directional fault current limiter and protection

11. Alam, M. N., Das, B. \& Pant, V. Protection coordination scheme for directional overcurrent relays considering change

12. Sarwagya, K., Nayak, P. K. \& Ranjan, S. Optimal coordination of directional overcurrent relays in complex distribution networks using sine cosine algorithm. Electr. Power Syst. Res. 187, 106435 (2020).

14. Aghdam, T. S., Karegar, H. K. \& Zeineldin, H. H. Transient stability constrained protection coordination for distribution systems with DG. IEEE Trans. Smart Grid 9, 5733-5741 (2017).

13. Khurshaid, T. et al. An improved optimal solution for the directional overcurrent relays coordination using hybridized whale optimization algorithm in complex power systems. IEEE Access 7, 90418-90435 (2019).

17. Jia, K., Yang, Z., Fang, Y., Bi, T. \& Sumner, M. Influence of inverter-interfaced renewable energy generators on directional relay and an improved scheme. IEEE Trans. power Electron. 34, 11843-11855 (2019).

18. Bozorgavari, S. A. et al. Robust planning of distributed battery energy storage systems in flexible smart distribution 
networks: A comprehensive study. Renew. Sustain. Energy Rev. 123, 109739 (2020).

414 19. Kavousi-Fard, A. \& Khodaei, A. Efficient integration of plug-in electric vehicles via reconfigurable microgrids. Energy 111, 653-663 (2016).

20. Norouzi, M., Aghaei, J. \& Pirouzi, S. Enhancing Distribution Network Indices Using Electric Spring under Renewable Generation Permission. in 2019 International Conference on Smart Energy Systems and Technologies (SEST) 1-6 (IEEE, 2019).

21. Pirouzi, S., Latify, M. A. \& Yousefi, G. R. Conjugate active and reactive power management in a smart distribution network through electric vehicles: A mixed integer-linear programming model. Sustain. Energy, Grids Networks 22, $100344(2020)$.

Hamidpour, H., Aghaei, J., Dehghan, S., Pirouzi, S. \& Niknam, T. Integrated resource expansion planning of wind integrated power systems considering demand response programmes. IET Renew. Power Gener. 13, 519-529 (2018).

Hamidpour, H., Aghaei, J., Pirouzi, S., Dehghan, S. \& Niknam, T. Flexible, reliable, and renewable power system resource expansion planning considering energy storage systems and demand response programs. IET Renew. Power Gener. 13, 1862-1872 (2019).

24. Seal, B. Interoperability, Information Exchange and Networks, and Protocols in IEEE Std 1547-2018. (2019).

25. Aghaei, J., Barani, M., Shafie-Khah, M., De La Nieta, A. A. S. \& Catalão, J. P. S. Risk-constrained offering strategy for aggregated hybrid power plant including wind power producer and demand response provider. IEEE Trans. Sustain. Energy 7, 513-525 (2015).

431 26. Mirjalili, S., Mirjalili, S. M. \& Lewis, A. Grey wolf optimizer. Adv. Eng. Softw. 69, 46-61 (2014).

432 27. Gill, H. S., Khehra, B. S., Singh, A. \& Kaur, L. Teaching-learning-based optimization algorithm to minimize cross entropy for Selecting multilevel threshold values. Egypt. Informatics J. 20, 11-25 (2019).

28. Babu, P. R., Rakesh, C. P., Kumar, M. N., Srikanth, G. \& Reddy, D. P. A novel approach for solving distribution networks. in 2009 Annual IEEE India Conference 1-5 (IEEE, 2009).

29. Ghasemi, S. \& Moshtagh, J. Radial distribution systems reconfiguration considering power losses cost and damage cost due to power supply interruption of consumers. Int. J. Electr. Eng. Informatics 5, (2013).

30. Norouzi, M., Aghaei, J., Pirouzi, S., Niknam, T. \& Lehtonen, M. Flexible operation of grid-connected microgrid using ES. IET Gener. Transm. Distrib. 14, 254-264 (2019).

31. Rani, R. R. \& Ramyachitra, D. Krill herd optimization algorithm for cancer feature selection and random forest technique for classification. in 2017 8th IEEE International Conference on Software Engineering and Service Science (ICSESS) 109-113 (IEEE, 2017). 
Figures

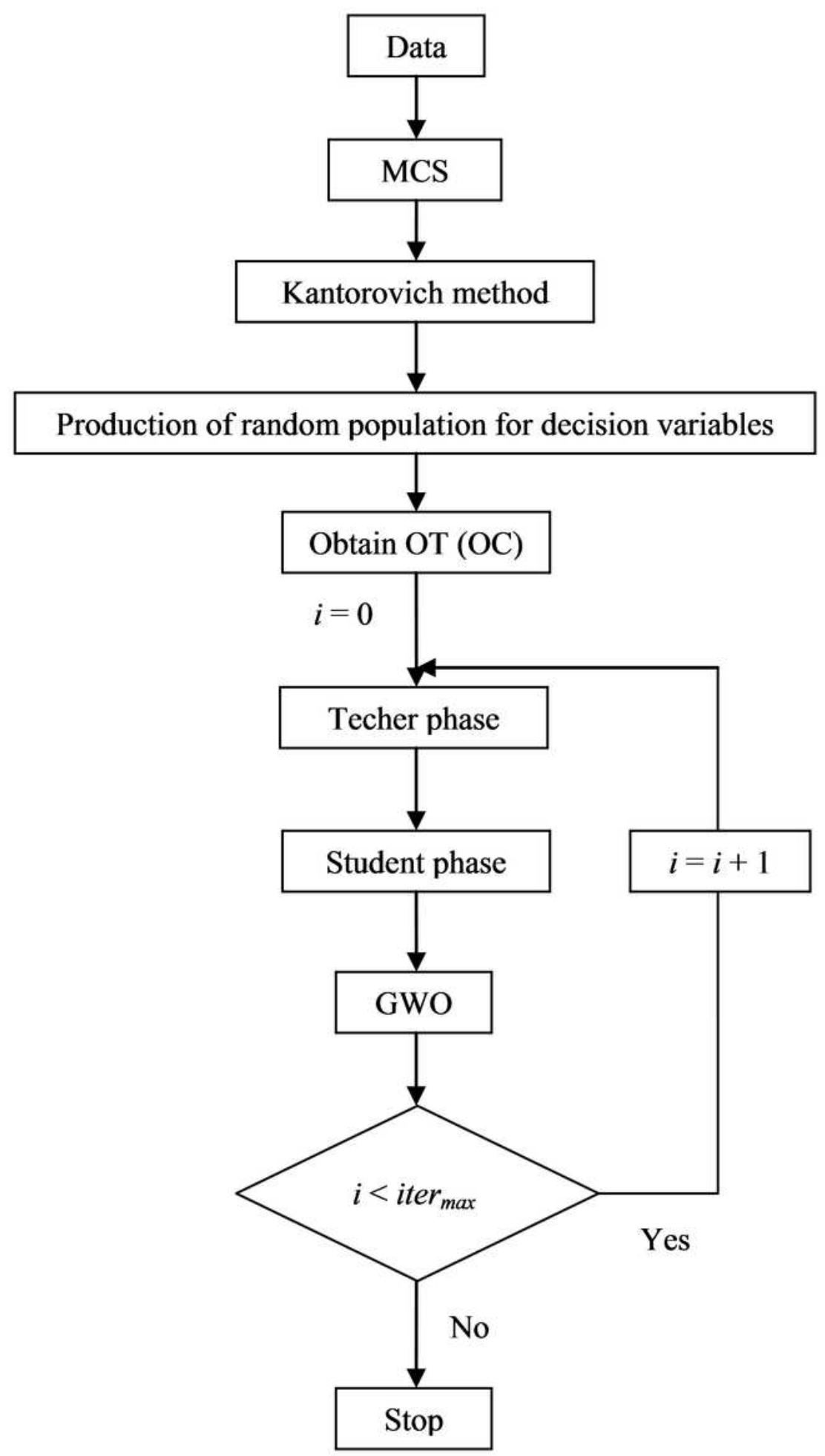

Figure 1

Flowchart of proposed solution algorithm 


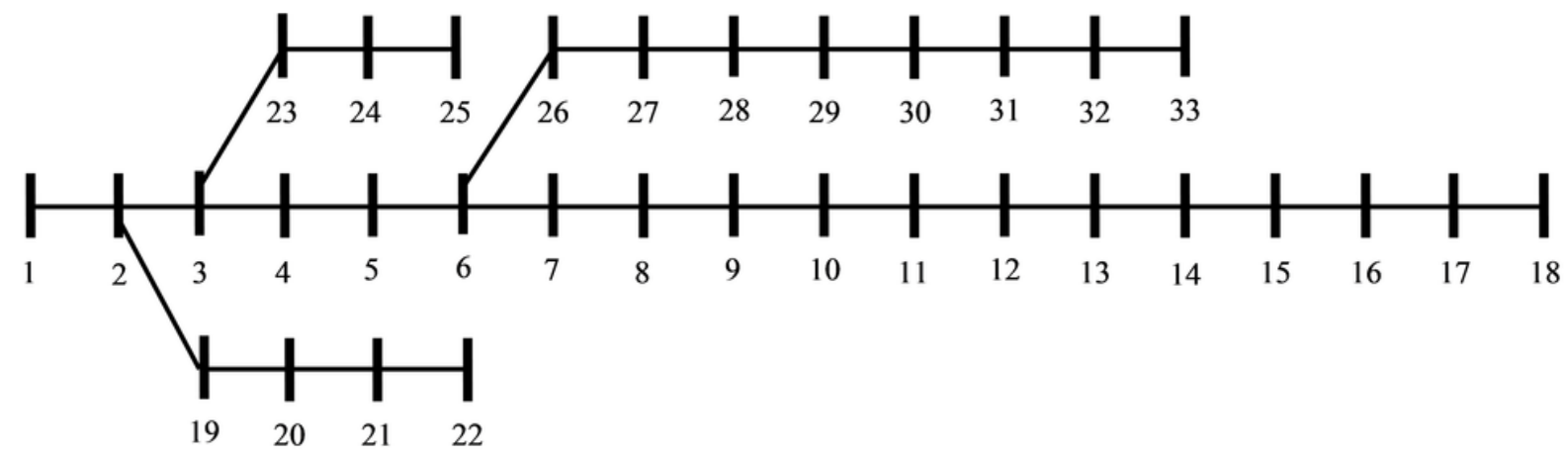

Figure 2

Single line diagram of IEEE 33-buses distribution network 29 


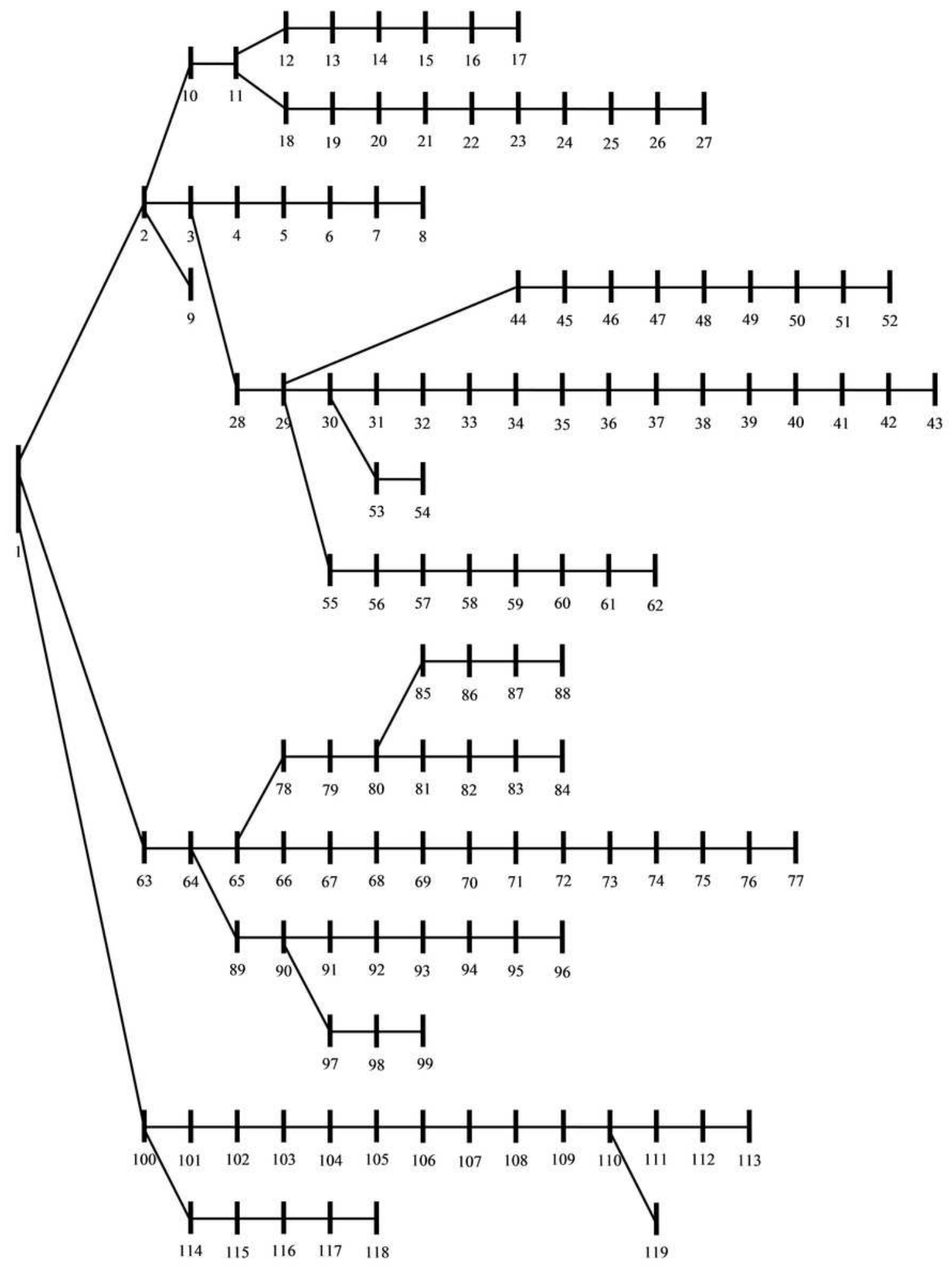

Figure 3

Single line diagram of IEEE 119-buses distribution network 29 


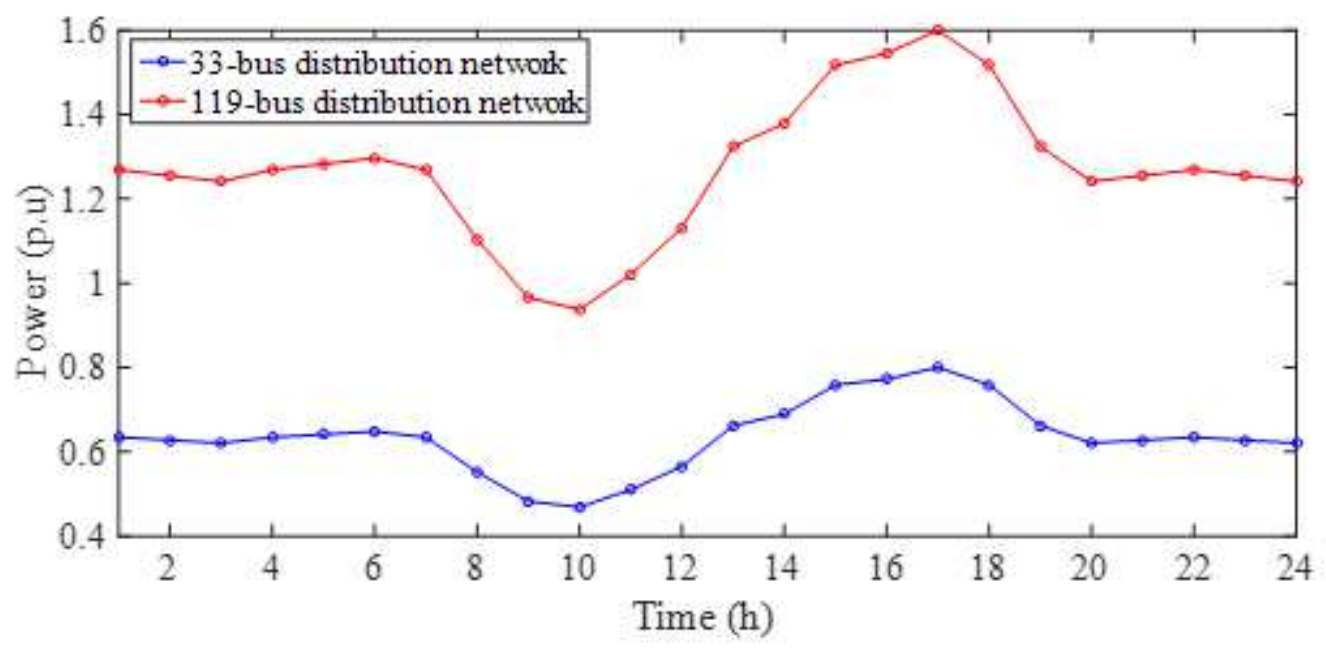

(a)

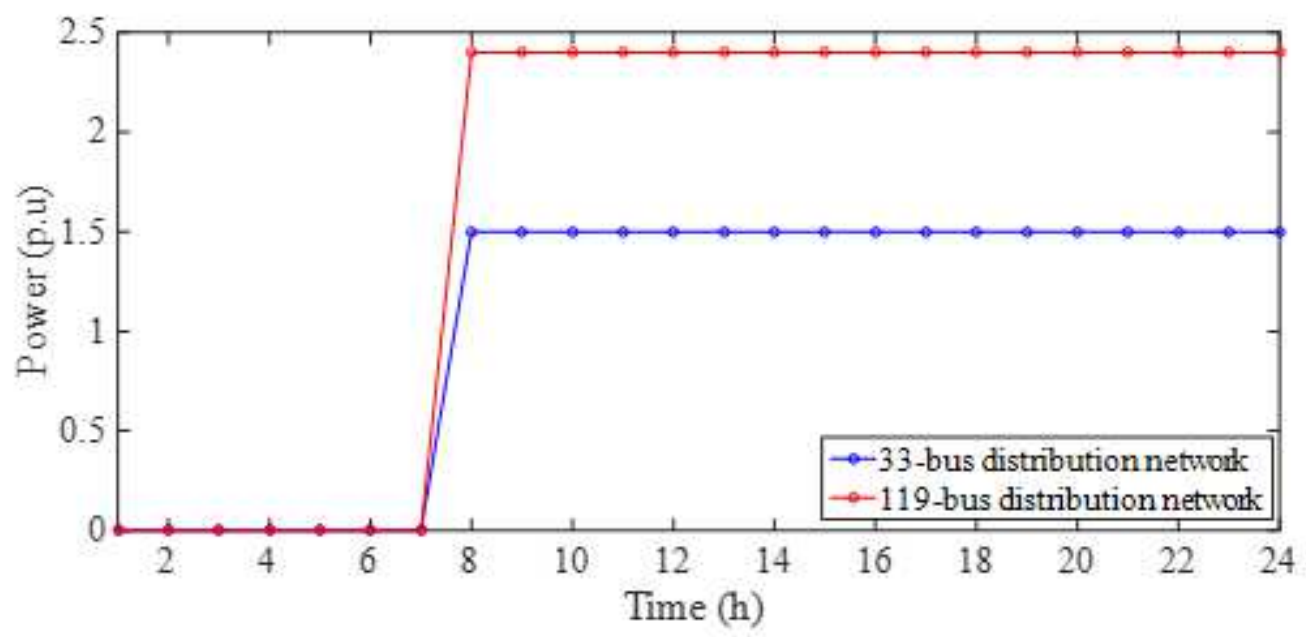

(b)

Figure 4

Expected active power daily curve, a) renewable SDGs, b) non-renewable SDGs 